
therapy for fibrovascular retinal pigment epithelial detachment due to occult age-related macular degeneration

Authors' Contribution:
A Study Design
B Data Collection
C Statistical Analysis
D Data Interpretation
E Manuscript Preparation
F Literature Search
G Funds Collection

Authors' Contribution:

D Data Interpretation

E Manuscript Preparation

G Funds Collection

\section{Małgorzata Figurska}

Department of Ophthalmology Military Institute of Medicine, Warsaw, Poland

Source of support: Departmental sources
Material/Methods:

Results:

Conclusions:

key words:

\title{
Summary
}

Background:

The aim of this paper is to report the incidence of retinal pigment epithelial (RPE) tears in patients treated with ranibizumab for subfoveal fibrovascular retinal pigment epithelial detachment (FVPED) due to occult age-related macular degeneration (AMD).

Thirty patients were treated according to the following schedule: saturation phase, further treatment was based on activity of the degeneration process. Visual acuity (VA), optical coherence tomography (OCT) and fluorescein angiography (FA) parameters were evaluated and compared.

Patients had a mean improvement of $+4.7 \pm 8.1$ letters at month 12 . The mean number of needed injections was $6.8 \pm 1.8$ (range, 3 to 9). RPE tears in fovea occurred in 8 cases (27\% of all patients). Analysis of variance revealed significant upper mean values of ETDRS letters for the subgroup without RPE tears. Mean values of PED height were significant upper for RPE tears without baseline. Statistical analysis revealed that in the subgroup without RPE tears mean values of VA significantly differed in succeeding periods compare to baseline $(\mathrm{P}<0.001)$. Visual improvement or stabilization was observed in $90.9 \%$ of patients without RPE tears (significant improvement of 15 or more letters in $22.7 \%-5 / 22$ ) and in $87.5 \%$ of patients with RPE tears (significant improvement was not observed). Baseline leakage parameters, lesion and leakage parameters at month 12 were significantly higher in patients with RPE tears. The chi-square test revealed statistically significant associations between RPE tears and subretinal fluid in OCT $(P<0.05)$ at month 12.

In eyes with FVPED and RPE tears treated with ranibizumab, stabilization of visual acuity without significant improvement is predictable. One of the risk factors common to RPE tears may be baseline leakage parameters and pretreatment distorted RPE contour in OCT. During ranibizumab therapy in eyes with RPE tears, upper parameters of FVPED height may occur without significant differences in fovea and macula volume compare to eyes without RPE tears.

retinal pigment epithelial tears • ranibizumab • occult exudative AMD

\section{Full-text PDF: \\ Word count: \\ Tables: \\ Figures: \\ References:}

Author's address:
http://www.medscimonit.com/fulltxt.php?ICID=882198

3673

3

2

26

Małgorzata Figurska Department of Ophthalmology Military Institute of Medicine, Szaserow 128 St., 04-141 Warsaw, Poland, e-mail: malgorzata-figurska@wp.pl 


\section{BACKGROUND}

Tears in the retinal pigment epithelium (RPE) occur spontaneously in the course of exudative age-related macular degeneration (AMD) [1,2]. RPE tears also occur following photodynamic therapy (PDT) and laser therapy, and are observed in polypoidal choroidal vasculopathy (PCV) [3-5]. Eyes with occult exudative AMD and retinal pigment epithelial detachment (PED) are especially predisposed to RPE tears [1,2]. RPE tears have been reported following intravitreal injections of vascular endothelial growth factor (VEGF) inhibitors, such as sodium pegaptanib, bevacizum$\mathrm{ab}$, and ranibizumab $[6,7]$.

The aim of this paper is to report the incidence of RPE tears in patients treated with ranibizumab (Lucentis ${ }^{\circledR}$, Novartis, Basel, Switzerland) for fibrovascular retinal pigment epithelial detachment (FVPED) due to occult age-related macular degeneration. Functional and morphological results of intravitreal ranibizumab injections and of FVPED treatment course during 12-month observation with the analysis a risk factors common to the RPE tears are presented.

\section{MATERIAL AND MethOdS}

After approval by the Bioethical Commission, 30 patients from the Retinal Clinic at the Department Of Ophthalmology of the Military Medical Institute in Warsaw were included in the study. Qualification criteria were as follows: 1 /age of over 50 years; 2/ subfoveal fibrovascular retinal pigment epithelial detachment with underlying active occult choroidal neovascularization $(\mathrm{CNV})$ confirmed on fluorescein angiography (FA) (Heidelberg Engineering HRA 2) and optical coherence tomography (SLO OCT OTI), not treated previously; 3/ overall lesion size not exceeding 12 optic disc diameters; 4/ baseline visual acuity (VA) of 0.2 logMAR-1.0 logMAR (34-75 letters of Early Treatment of Diabetic Retinopathy Study - ETDRS chart). Patients with the following conditions and past treatments were not qualified for ranibizumab treatment: 1/ permanent structural fovea damage (subretinal scar or geographic atrophy); 2/ macular hemorrhage exceeding $50 \%$ of lesion size; 3 / retinal detachment; 4 / vitreoretinal or filtration surgery, or transplantation of the cornea; 5 / peripheral retinal photocoagulations within the last month; 6 / macular photocoagulations; 7 / cataract surgery within the last 2 months; 8 / unstabilized glaucoma; 9/ active infection of the eyeball or its protective apparatus; 10 / past or active uveitis; 11/ significant degeneration of peripheral retina. The most important general exclusion criteria were cerebral stroke or myocardial infarction within the last 6 months, severe unstabilized hypertension, and unstable coronary heart disease.

Mean age was $74.46 \pm 7.75$ years (61-86 years); 19 women and 11 men were treated. Treatment was performed according to the following schedule: each patient received 3 intravitreal injections of $0.5 \mathrm{mg}$ ranibizumab in monthly intervals (saturation phase); further treatment was based on activity of the exudative degeneration process. After the end of the saturation phase, each month the patients' VA was tested with the ETDRS chart and OCT was performed. The following re-injection criteria were employed: $1 /$ loss of 5 or more ETDRS letters compared to best result during the initial phase of treatment with OCT evidence of fluid in the macula; 2/ persistence or appearance of fluid under the retina or intraretinal edema, enlargement of PED on OCT; 3 / increase in central retinal point thickness of at least $100 \mu \mathrm{m}$ compared to lowest value during saturation phase; 4/ new macular hemorrhage; 5 / new CNV focus on FA. Each ranibizumab injection was performed in an operation theatre with aseptic rules. Before and after the injection a broad-spectrum antibiotic was being administered for 3 days. During treatment the patients were being monitored for topical and general adverse events and the number of needed ranibizumab injections was counted.

Baseline, 1 month after the $1^{\text {st }}, 2^{\text {nd }}$ and $3^{\text {rd }}$ ranibizumab injection and at 12 months, VA (ETDRS letters) and OCT parameters (central retinal point thickness CRPT- $\mu \mathrm{m}$, fovea volume $F V-\mathrm{mm}^{3}$, total macular volume $T M V-\mathrm{mm}^{3}$ from 3D Retinal Topography full field $29.2^{\circ}$ scans, and PED height $P E D H-\mu m$, PED base $P E D B$ greatest linear diameter- $\mu \mathrm{m}$ from $6 \mathrm{~mm}$ radial scans, manual measurements) were evaluated and compared. 3D Retinal Topography was performed with option high-resolution frames, high-quality (3s), high-speed (1s) with scan depth $2.3 \mathrm{~mm}$. Data were analyzed (option ANALYZE) and presented as a 3-D topography map of retinal thickness measured between RPE and vitreus surface. In zone analysis by SLO OCT OTI retinal thickness and volume were evaluated: central point retinal thickness, volume of central circle $1 \mathrm{~mm}$ diameter (fovea), volume of central circle $6 \mathrm{~mm}$ diameter (total macula). Radial scans were performed with option zoom radial, radial increments 15 degrees and scan depth $2.3 \mathrm{~mm}$. For PED base measurement, RPE line was prolonged and section between acute detachment borders was evaluated. For PED height measurement, perpendicular line to the RPE was conducted and section between the highest point of PED and base was evaluated. Select radial scans from succeeding periods are compared (option COMPARE).

FA was performed at baseline and at month 12. Lesion (LMPS) and leakage (LEMPS) size in the Macular Photocoagulation Study Group disk area were evaluated and compared. Each of the eligible patient's baselines had a PED with subretinal fluid $(S F)$ in OCT as a one of the qualification criteria. At one month after the $3^{\text {rd }}$ ranibizumab injection and at 12 months, presence of SF was evaluated and compared.

After 12 months the results of this therapeutic approach were summed up. To compare mean values of visual acuity, OCT parameters at the 5 points included in the study (baseline, after the $1^{\text {st }}, 2^{\text {nd }}$ and $3^{\text {rd }}$ injection, and at month 12 of treatment) and FA mean values parameters at the 2 points (baseline and at 12 months), a Student's T test was used. For many variables, analysis of variance with ANOVA and least significant differences (LSD) tests were performed or Friedman's ANOVA, depending whether the variables had normal distribution. To compare values between subgroups, the Student's T test or the Mann-Whitney $\mathrm{U}$ test were used. The associations between the RPE tears and $\mathrm{SF}$ were assessed using a chi-square test. Statistical significance was defined as $P$ values $<0.05$. All analyses were performed using Statistica 9.2 program (StatSoft, Poland).

\section{RESULTS}

Statistical analysis showed that mean values of VA (ETDRS letters) were significantly different in succeeding intervals 
and began to improve after the first ranibizumab injection. Mean best corrected visual acuity (BCVA) at baseline was $53.27 \pm 11.52$ ETDRS letters, after the first injection was $57.33 \pm 12.35$, and after the third injection was $60.60 \pm 13.10$. The improvement was sustained and at month 12 mean VA was $58.00 \pm 12.20$ letters. Patients had a mean improvement of $+4.7 \pm 8.1$ letters at month 12 . Visual improvement of 15 or more letters was observed in $16.7 \%$ (5/30) of patients. Visual improvement or stabilization was observed in $90 \%(27 / 30)$ of patients. Three patients (10\%) lost 5 or more letters.

Statistical analysis showed that mean values of CRPT, FV and TMV significantly differed in succeeding periods and began decreasing after the first ranibizumab injection. Mean CRPT at baseline was $354.80 \pm 159.39 \mu \mathrm{m}$, after the first injection $289.23 \pm 113.80 \mu \mathrm{m}$, after the third injection $205.67 \pm 65.38 \mu \mathrm{m}$, and after 12 months $225.67 \pm 72.77 \mu \mathrm{m}$. Frequencies of eyes with CRPT >200 $\mu$ m were: baseline $100 \%, 1$ month $80 \%, 3$ months $57 \%$, and 12 months $73 \%$. Mean FV at baseline was $0.26 \pm 0.10 \mathrm{~mm}^{3}$, after the first injection $0.21 \pm 0.05 \mathrm{~mm}^{3}$, after the third injection $0.18 \pm 0.04 \mathrm{~mm}^{3}$, and after 12 months $0.19 \pm 0.067 \mathrm{~mm}^{3}$. Mean TMV at baseline was $8.80 \pm 2.27 \mathrm{~mm}^{3}$, after the first injection $8.010 \pm 1.398 \mathrm{~mm}^{3}$, after the third injection $7.22 \pm 0.88 \mathrm{~mm}^{3}$, and after 12 months $7.35 \pm 1.24 \mathrm{~mm}^{3}$.

Mean values of PEDB were significantly different after the second injection and in succeeding periods compare to baseline. After 12 months there was a significant different in PEDB measurements $(3503.23 \pm 1143.67$ vs. $3089.13 \pm 1347.23$ $\mu \mathrm{m})$. Mean values of PEDH were significantly different after the second injection and in succeeding periods compared to baseline. Mean PEDH at baseline was $460.53 \pm 159.69$ and after 12 months it decreased to $345.60 \pm 191.61 \mu \mathrm{m}$.

Mean values of FA parameters were significantly different at baseline and at month 12. Mean LMPS at baseline was $2.99 \pm 0.84$ DD and $2.73 \pm 0.89$ DD after 12 months. Mean LEMPS at baseline was $1.63 \pm 0.60 \mathrm{DD}$ and $1.32 \pm 0.79 \mathrm{DD}$ after 12 months.

The total number of ranibizumab injections during 12 months of observation was 203. The mean number of injections needed was $6.8 \pm 1.8$ (range, 3 to 9 ). After the third injection, SF was observed in 10 cases ( $33 \%$ of all patients) and after 12 months in 13 cases (43\% of all patients).

RPE tears occurred in 8 cases (27\% of all patients) and were the main ocular adverse events during the present study, in addition to fovea atrophy in 1 patient with visual loss of 13 letters. In 6 cases, RPE tears were detected after the first ranibizumab injection, and in 2 cases during saturation phase. Two patients with RPE tears reported greater image distortion; the others did not observe this symptom. In all RPE tears cases, a grayish, curled pigment epithelium was observed in the central macular area. OCT confirmed RPE tears involving fovea in all cases. One patient after saturation phase refused consecutive ranibizumab injection because of significant visual deterioration, but was observed, and OCT and VA were monitored.

Statistical analysis showed significant differences between the subgroup of 22 patients without RPE tears and the subgroup of 8 patients with RPE tears at the consecutive time intervals in mean values of ETDRS, LEMPS at baseline,
ETDRS after the $1^{\text {st }}, 2^{\text {nd }}, 3^{\text {rd }}$ injection, and after 12 months, LMPS, LEMPS after 12 months, PEDH after the $1^{\text {st }}, 2^{\text {nd }}, 3^{\text {rd }}$ injection and 12 months (Tables 1-3). There were no significant differences between mean number of ranibizumab injections $7.1 \pm 1.8$ vs. 5.9 \pm 1.5 , PEDB parameters (baseline $3408.14 \pm 1053.37$ vs. $3764.75 \pm 1408.30 \mu \mathrm{m} P=0.46$, after 12 months $3106.18 \pm 1454.46$ vs. $3042.25 \pm 1081.52 \mu \mathrm{m} P=0.91$ ), and baseline PEDH (431. $41 \pm 139.87$ vs. $540.62 \pm 192.24 \mu \mathrm{m}$, $P=0.10)$.

Analysis of variance revealed significant differences in time course for mean values of ETDRS letters $(P<0.05)$ (Figure1) with significant upper parameters for patients without RPE tears, and PED height $(P<0.05)$ with significant upper parameters for RPE tears without baseline (Figure 2). There were no significant differences in time course for median values of CRPT, FV, TMV, and mean PEDB. The mean improvement of $+6.0 \pm 8.1$ letters at 12 months was observed in the subgroup without RPE tears, and of $+1.3 \pm 7.5$ letters in the RPE tears subgroup without significant difference $(P=0.16)$. LSD test revealed that in the subgroup without RPE tears, mean values of VA significantly differed in succeeding periods compared to baseline $(\mathrm{P}<0.001)$. In the subgroup with RPE tears, significant differences in mean values of VA were not observed. Visual improvement or stabilization was observed in $90.9 \%(20 / 22)$ of patients without RPE tears (improvement of 15 or more letters in $22.7 \%$ $-5 / 22)$. Two patients $(9.1 \%)$ lost 5 or more letters. Visual improvement or stabilization was observed in $87.5 \%(7 / 8)$ of patients with RPE tears (significant improvement was not observed). One patient (12.5\%) lost 12 letters.

The chi-square test revealed statistically significant associations between RPE tears and SF in OCT at month 12 $(P<0.05)$. No topical or general adverse events were observed.

\section{DISCUSSION}

In the era of anti-VEGF therapy in patients with exudative $\mathrm{AMD}$, there has been a resurgence of new publications about RPE tears [8]. RPE tears may occur spontaneously in the course of exudative AMD. Casswell et al. [1] reported RPE tears in $10 \%$ of eyes with PED during a 5-year observation period. Pauleikhoff et al. [2] reported RPE tears in $12.5 \%$ of eyes with vascular PED after 10.7 months. A report by Lee et al on RPE tears following ranibizumab therapy is significant in the context of this research [7]. The authors presented the case of a 70-year-old patient with FVPED who had been treated with 3 sodium pegaptanib injections. Because of the observed increase in PED and persistent leakage from the CNV, the patient was switched to ranibizumab therapy. One day following the third ranibizumab dose, RPE tears in the fovea occurred. There was no tear progression and the ranibizumab therapy was withdrawn. In a 3-month follow-up after the injection complicated by tears, visual acuity was stable at the 20/100 level. In this author's observation, 6 cases of RPE tears were detected after the first ranibizumab injection, and 2 cases during the saturation phase. The timing of the tear onset (within the first month after the injection) suggests a correlation with the effects of the medication. Other reports confirm this correlation, although reports of the onset of tears 4 to 6 weeks following bevacizumab injections predominate in the literature [9-12]. Kiss et al. described 2 cases of RPE tears after intravitreal injections of 
Table 1. Visual acuity (number of ETDRS letters) significant differences between subgroup of 22 patients without RPE tears and subgroup of 8 patients with RPE tears at the consecutive time intervals (the Student's t-test).

\begin{tabular}{|c|c|c|c|c|c|c|c|c|}
\hline & $\begin{array}{c}\text { Mean } \\
\text { values } \\
\text { without } \\
\text { RPE tears } \\
22 \text { eyes }\end{array}$ & $\begin{array}{c}\text { Mean } \\
\text { values } \\
\text { with } \\
\text { RPE tears } \\
8 \text { eyes }\end{array}$ & $\begin{array}{c}\text { Standard } \\
\text { deviation } \\
\text { without } \\
\text { RPE tears }\end{array}$ & $\begin{array}{l}\text { Standard } \\
\text { deviation } \\
\text { with } \\
\text { RPE tears }\end{array}$ & $\begin{array}{l}\text { Standard } \\
\text { error } \\
\text { without } \\
\text { RPE tears }\end{array}$ & $\begin{array}{l}\text { Standard } \\
\text { error } \\
\text { with } \\
\text { RPE tears }\end{array}$ & $\mathbf{t}$ & $\mathbf{p}$ \\
\hline Baseline ETDRS & 56.1 & 45.6 & 10.0 & 12.5 & 2.1 & 4.4 & 2.4 & $<0.05$ \\
\hline ETDRS after the $1^{\text {st }}$ injection & 61.6 & 45.5 & 9.3 & 12.4 & 1.9 & 4.4 & 3.8 & $<0.001$ \\
\hline ETDRS after the $2^{\text {nd }}$ injection & 63.9 & 47.0 & 9.4 & 11.5 & 2.0 & 4.1 & 4.1 & $<0.001$ \\
\hline ETDRS after the $3^{\text {rd }}$ injection & 65.4 & 47.5 & 10.0 & 12.1 & 2.1 & 4.3 & 4.1 & $<0.001$ \\
\hline ETDRS after 12 months & 62.1 & 46.9 & 10.1 & 10.7 & 2.2 & 3.8 & 2.9 & $<0.05$ \\
\hline
\end{tabular}

Table 2. OCT significant differences between subgroup of 22 patients without RPE tears and subgroup of 8 patients with RPE tears at the consecutive time intervals (the Student's t-test).

\begin{tabular}{|c|c|c|c|c|c|c|c|c|}
\hline & $\begin{array}{c}\text { Mean } \\
\text { values } \\
\text { without } \\
\text { RPE tears } \\
22 \text { eyes }\end{array}$ & $\begin{array}{c}\text { Mean } \\
\text { values } \\
\text { with } \\
\text { RPE tears } \\
8 \text { eyes }\end{array}$ & $\begin{array}{c}\text { Standard } \\
\text { deviation } \\
\text { without } \\
\text { RPE tears }\end{array}$ & $\begin{array}{c}\text { Standard } \\
\text { deviation } \\
\text { with RPE } \\
\text { tears }\end{array}$ & $\begin{array}{l}\text { Standard } \\
\text { error } \\
\text { without } \\
\text { RPE tears }\end{array}$ & $\begin{array}{l}\text { Standard } \\
\text { error } \\
\text { with } \\
\text { RPE tears }\end{array}$ & $\mathbf{t}$ & p \\
\hline Baseline PEDH & 431.4 & 540.6 & 139.9 & 192.2 & 29.8 & 68.0 & -1.7 & $P=0.10$ \\
\hline PEDH after the $1^{\text {st }}$ injection & 390.2 & 585.4 & 164.2 & 164.5 & 35.0 & 58.1 & -2.9 & $<0.05$ \\
\hline PEDH after the $2^{\text {nd }}$ injection & 294.4 & 520.5 & 121.3 & 174.8 & 25.9 & 61.8 & -4.0 & $<0.001$ \\
\hline PEDH after the $3^{\text {rd }}$ injection & 263.1 & 507.4 & 154.3 & 183.3 & 32.9 & 64.8 & -3.6 & $<0.05$ \\
\hline PEDH after 12 months & 275.3 & 538.9 & 145.6 & 173.9 & 31.1 & 61.5 & -4.2 & $<0.001$ \\
\hline
\end{tabular}

PEDH - pigment pithelium detachment height $(\mu \mathrm{m})$.

Table 3. Fluorescein angiography significant differences between subgroup of 22 patients without RPE tears and subgroup of 8 patients with RPE tears at the consecutive time intervals (the Student's t-test).

\begin{tabular}{lccccccccc}
\hline & $\begin{array}{c}\text { Mean } \\
\text { values } \\
\text { without } \\
\text { RPE tears } \\
\text { 22 eyes }\end{array}$ & $\begin{array}{c}\text { Mean } \\
\text { values } \\
\text { with RPE } \\
\text { tears } \\
\mathbf{8} \text { eyes }\end{array}$ & $\begin{array}{c}\text { Standard } \\
\text { deviation } \\
\text { without } \\
\text { RPE tears }\end{array}$ & $\begin{array}{c}\text { Standard } \\
\text { deviation } \\
\text { with } \\
\text { RPE tears }\end{array}$ & $\begin{array}{c}\text { Standard } \\
\text { error } \\
\text { without } \\
\text { RPE tears }\end{array}$ & $\begin{array}{c}\text { Standard } \\
\text { error } \\
\text { with RPE } \\
\text { tears }\end{array}$ & t & p \\
\hline Baseline LEMPS & 1.5 & 2.0 & 0.6 & 0.4 & 0.1 & 0.1 & -2.3 & $<0.05$ \\
\hline LMPS after 12 months & 2.5 & 3.4 & 0.9 & 0.6 & 0.2 & 0.2 & -2.6 & $<0.05$ \\
\hline LEMPS after 12 months & 1.0 & 2.1 & 0.6 & 0.7 & 0.1 & 0.2 & -4.3 & $<0.001$ \\
\hline
\end{tabular}

LEMPS - leakage size in MPSG disk area; LMPS - lesion size in MPSG disk area.

ranibizumab in patients with neovascular AMD [13]. One patient developed the RPE tears within 2 weeks of the injection, the other within 6 weeks of a second injection. Both patients presented vision loss of 1 line at diagnosis of RPE damage, but during long-term follow-up, visual acuity improved in 1 patient by 1 line and deteriorated in the second patient by 3 lines. The authors suggested that the rapid regression of the fibrovascular membrane promote RPE tears. In the present study, a significant visual deterioration (loss of 12 letters) was observed in 1 patient with RPE tears. In the others $(87.5 \%)$, stabilization or visual improvement of up to 13 letters in 1 case were noted. The significant differences in mean values of VA did not occur during the 12-month observation (LSD test).

Smith et al. reported only 1 incidence $(0.61 \%)$ of RPE tears in a ranibizumab-treated group of 164 patients with wet 


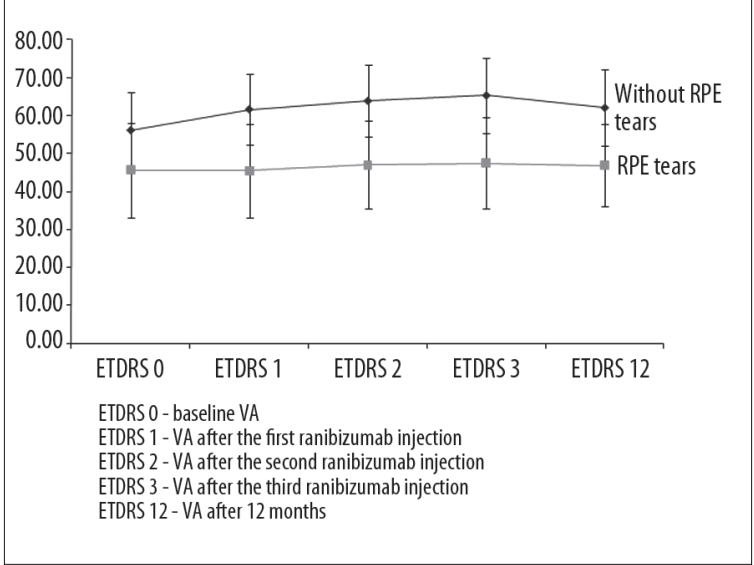

Figure 1. Graph of changes in mean VA (in number of ETDRS letters) in consecutive periods (baseline, after $1^{\text {st }}, 2^{\text {nd }}, 3^{\text {rd }}$ injection, after 12 months) during ranibizumab therapy.

AMD (occult lesions 60\%, subfoveal location 68\%, FVPED 19 eyes $-12 \%$ ) [14]. This occurred in a 78-year-old female with FVPED ( $5 \%$ of eyes with FVPED, $1 \%$ of eyes with occult CNV, 1/105 injections in eyes with a FVPED $-0.95 \%$ ) with a greatest linear diameter of $3254 \mu \mathrm{m}$, who returned 4 weeks after ranibizumab injection with visual improvement from $20 / 200$ to $20 / 100$. She has remained stable over 12 months without further injections. In this interventional case series, the occult lesions containing a FVPED tended to be larger (4.5 MPS DA compared with 3.8), and FVPED eyes required 6 injections on average, more than the average for the entire cohort. Additionally, there was an average decrease in vision with a FVPED (-0.15 logMAR) compared with the entire cohort $(0.05 \operatorname{logMAR})$ with statistically significant difference. Smith et al. described in their conclusion that incidence of RPE tears associated with ranibizumab therapy is low and may result from a predisposition (FVPED) rather than being an effect of treatment. In the present study, RPE tears occurred in $27 \%$ of all FVPED patients and this is a high value. There were no significant differences between numbers of ranibizumab injections $6.8 \pm 1.8$ for all groups vs. $5.9 \pm 1.5$ for the RPE tears subgroup. Visual stabilization or improvement was observed in $90 \%$ of patients, with a mean improvement of $+4.7 \pm 8.1$ letters at 12 months. The mean improvement of $+6.0 \pm 8.1$ letters at 12 months was observed in the subgroup without RPE, and of $+1.3 \pm 7.5$ letters in the RPE tears subgroup, but without significant difference $(P=0.16)$. However, in time course mean values of VA parameters were significantly lower for RPE tears.

Lommatzsch et al. retrospectively analyzed a group of 328 patients with serous PED and documented visual deterioration treated with anti-VEGF agents, PDT with steroids [15]. In spite of visual acuity improvement by about 0.066 logMAR and retinal thickness reduction in all patients, 41 $(12.5 \%)$ developed RPE tears. In the authors' opinion, tears of RPE or only partial flattening of the PED always indicated a worse prognosis in eyes with exudative AMD than in eyes with classic choroidal neovascularization. RPE tears are an extremely rare complication in patients with classic $\mathrm{CNV}$ without RPE detachment. Konstantinidis et al. observed RPE tears in $4(5.4 \%)$ eyes after a mean of 4 ranibizumab injections [16]. Mean baseline BCVA was $0.67 \mathrm{log}$

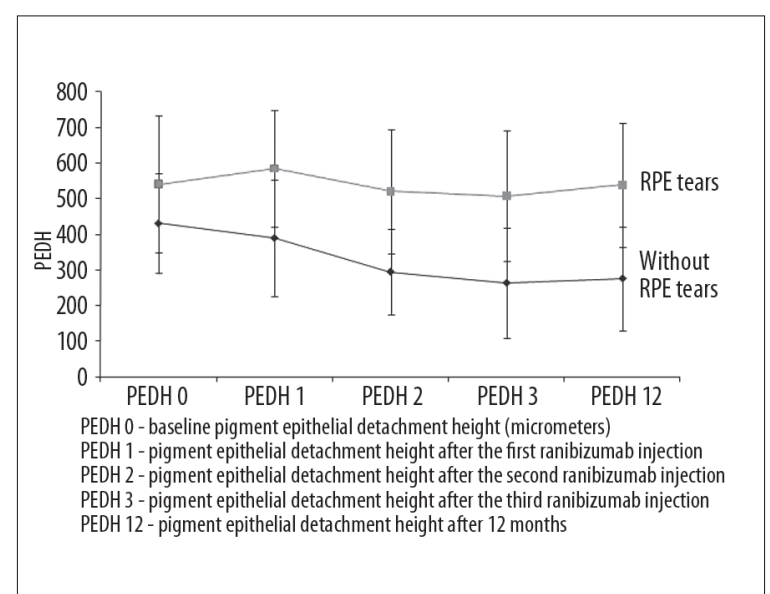

Figure 2. Graph of changes in mean VA (in decimal scale results) in consecutive periods (baseline, after $1^{\text {st }}, 2^{\text {nd }}, 3^{\text {rd }}$ injection, after 12 months) during ranibizumab therapy.

MAR and improved despite the RPE tear to $0.22 \log$ MAR. Arias et al. reported 3 cases of RPE tears in a group of 40 patients with predominantly classic CNV secondary to AMD who were treated with $1.25 \mathrm{mg}$ of intravitreal bevacizumab [17]. RPE tears developed after the first injection. Arias et al. found that in cases with RPE tears VA is not always affected and is conditioned by localization of RPE damage (in the present study all RPE tears involved fovea).

The pathogenesis of RPE tears is not completely understood. It is most frequently explained by translation of the $\mathrm{CNV}$ forces on the RPE or the effect of hydrostatic pressure in the space under the detached RPE. Rapid absorption of the fluid underneath the RPE appears to be a coexisting factor [8]. According to Gass, RPE tears are formed as a result of a rapid loss of vascular integrity, which results in an abrupt PED enlargement [18]. Lee et al. emphasized the role of the traction force associated with the CNV expansion or shrinkage of the CNV/RPE complex as a result of VEGF inhibitor activity [7]. Krishan et al. also pointed to the tangents of the forces acting during contraction of the subretinal membrane as the cause of tears [19]. Recurring subretinal fluid due to breakdown of the outer blood-retinal barrier following interruption and folding of the RPE was observed in the patients, despite transient positive visual effects (the study revealed statistically significant associations between RPE tears and SF).

Because RPE tears are a significant clinical problem, retina specialists must determine the risk factors. Leitritz et al. [20] performed a retrospective evaluation of OCT of 393 eyes with CNV (Stratus-OCT Zeiss, Jena, Germany). The height of the PED that accompanied CNV, thickness of the central retina, and maximal thickness of the retina were measured. In the 15 eyes in which RPE tears occurred following bevacizumab administration, the PED was significantly higher than in the other group. There was no positive correlation between the preprocedural thickness of the central retina, the maximal thickness of the retina, and the accompanying intraretinal swelling. There was a strong correlation between the PED height and the overall size of the lesion, as well as with the size of the CNV focal lesion. The risk for RPE tears increased from $0.5 \%(100 \mu \mathrm{m})$ to $14.8 \%$ 
$(600 \mu \mathrm{m})$ with an increase in the RPE detachment height. In the absence of PED prior to the procedure, the risk of RPE tears was $0.3 \%$. The present study revealed significant differences in time course for mean values of PED height $(P<0.001)$ with significant upper parameters for RPE tears, but without significant differences in baseline values, fovea and macula volume compared to eyes without RPE tears.

Chan et al. observed RPE tears in 21 FVPED eyes among 125 FVPED eyes (16.8\% of eyes with FVPED and 2.1\% of all analyzed eyes) after bevacizumab injections [21]. The mean time interval from the bevacizumab injection to the development of an RPE tear was $30.4 \pm 22.7$ days. Significantly greater mean FVPED height $(648.9 \pm 245 \mu \mathrm{m})$ with more subretinal fluid was found in the RPE tears subgroup in comparison to the FVPED subgroup without RPE damage $(338.1 \pm 201.6$ $\mu \mathrm{m})$. Also, in the RPE tears subgroup there was higher mean FVPED volume index $\left(9.3 \pm 6.3\right.$ vs. $\left.3.5 \pm 4.0 \mathrm{~mm}^{3}\right)$ and total mean macular volume $\left(8.0 \pm 1.8\right.$ vs. $\left.7.4 \pm 1.2 \mathrm{~mm}^{3}\right)$. The present study did not reveal significant differences in FV and TMV between subgroup parameters.

Gelisken et al. reported RPE tears in $3.6 \%$ of eyes $(15 / 409)$ following a single administration of bevacizumab [22]. The exudative lesions classified as overall medium (between 4 and 6 disc diameters, DD) and large (more than 6 DD) had a statistically higher tear incidence. If subretinal hemorrhage was predominant in the entire lesion, the tear incidence was $6.9 \%$. In the present study baseline leakage parameters in FA were significantly different in patients with RPE tears $(P=0.03)$, without significant difference in baseline lesions parameters.

The initial condition of the contour of the detached pigment epithelium is important for predicting RPE tears, as pointed out by Moroz et al., who conducted a retrospective evaluation of the course of treatment with bevacizumab in 24 eyes in which serous PED was originally diagnosed [23]. In 6 eyes, RPE tears occurred shortly after the first injection. Two typical reproducible phenomena were observed in the eyes in which tears developed. The first was multifocal corrugations and waves with small elevations of the RPE. The second was step-like interruptions or interruptions of the continuity of the RPE line. One of these phenomena was observed in OCT prior to bevacizumab administration in every eye in which RPE tears later developed. In the group of 18 eyes in which RPE tears did not develop, corrugation was observed in two eyes and steplike microinterruptions were not observed at all. The differences were statistically significant. Among patients with RPE tears from the present study, 4 had interruptions of the RPE line at baseline OCT. Shiraki et al. [24] described a case of spontaneous RPE tears. In earlier OCT, small interruptions of the RPE line were observed. These interruptions were considered to be microholes in the RPE layer and not full thickness tears.

\section{Conclusions}

VEGF is currently considered the most important mediator of angiogenesis in physiological and pathological processes, including various eye diseases such as exudative AMD $[25,26]$. The preferred method of neovascularization treatment is intravitreal administration of VEGF inhibitors.
Various preparations, including off-label therapies, such as bevacizumab, are used. Intravitreal administration of ranibizumab is recommended as the first treatment. Isolated cases of RPE tears following ranibizumab administration are described, and the treatment seems safe. The available literature primarily refers to therapy with bevacizumab. Significant risk factors for RPE tears following ranibizumab and bevacizumab injections, such as occult CNV with PED, have been identified and confirmed. This author's experience demonstrates that in eyes with FVPED and RPE tears treated with ranibizumab, stabilization of visual acuity without significant improvement is predictable. One of the risk factors common to RPE tears may be baseline leakage parameter and pretreatment distorted RPE contour in OCT. During ranibizumab therapy in eyes with RPE tears, upper parameters of FVPED height may occur without significant differences in fovea and macula volume compared to eyes without RPE tears. Nevertheless, tears may cause bothersome image distortions and substantial discomfort, as emphasized by patients. It should be kept in mind that tears may also be generated spontaneously in exudative AMD. Prior to initiating anti-VEGF therapy, known and confirmed RPE risk factors should be considered and discussed with patients at risk as part of the pre-operative evaluation.

\section{REFERENCES:}

1. Casswell AG, Kohen D, Bird AC: Retinal pigment epithelial detachments in elderly: classification and outcome. Br J Ophthalmol, 1985; 69: 397-403

2. Pauleikhoff D, Loeffert D, Spital G et al: Pigment epithelial detachment in elderly. Clinical differentiation, natural course and pathogenetic implications. Graefes Arch Clin Exp Ophthalmol, 2002; 533-38

3. Goldstein M, Heilweil G, Barak A, Loewenstein A: Retinal pigment epithelial tear following photodynamic therapy for choroidal neovascularization secondary to AMD. Eye, 2005; 19: 1315-24

4. Torron-Fernandez-Blanco C, Ferrer-Novella E, Moreno-Ruiz O, HonrubiaLopez FM: Optical coherency tomography of retinal pigment epithelial tears. Arch Soc Esp Oftalmol, 2007; 82: 245-50

5. Tsujikawa A, Hirami Y, Nakanishi $\mathrm{H}$ et al: Retinal pigment epithelial tear in polypoidal choroidal vasculopathy. Retina, 2007; 27: 832-38

6. Dhalla MS, Blinder KJ, Tewari A et al: Retinal pigment epithelial tear following intravitreal pegaptanib sodium. Am J Ophthalmol, 2006; 141: $752-54$

7. Lee GKY, Lai TYY, Chan LWM, Lam DSC: Retinal epithelial tear following intravitreal ranibizumab injections for neovascular age-related macular degeneration. Graefe's Arch Clin Exp Ophthalmol, 2007; 245: 1222-27

8. Chang LK, Sarraf D: Tears of the retinal pigment epithelium. An old problem in a new era. Retina, 2007; 27: 523-34

9. Gelisken F, Ziemssen F, Voelker M, Bartz-Schmidt KU: Retinal pigment epithelial tear following intravitreal bevacizumab injection for neovascular age-related macular degeneration. Acta Ophthalmol Scand, 2006; 84: 833-34

10. Bakri SJ, Kitzmann AS: Retinal pigment epithelial tear after intravitreal ranibizumab. Am J Ophthalmol, 2007; 143: 505-7

11. Carvounis PE, Kopel AC, Benz MS: Retinal epithelium tears following ranibizumab for exudative age-related macular degeneration. Am J Ophthalmol, 2007; 143: 504-5

12. Shah CP, Hsu J, Garg SJ et al: Retinal pigment epithelial tear after intravitreal bevacizumab injection. Am J Ophthalmol, 2006; 142: 1070-72

13. Kiss C, Michels S, Prager F et al: Retinal pigment epithelium tears following intravitreal ranibizumab therapy. Acta Ophthalmol Scand, 2007; 85: 902-3

14. Smith BT, Kraus CL, Apte RS: Retinal pigment epithelial tears in ranibizumab-treated eyes. Retina, 2009; 29: 335-39

15. Lommatzsch A, Heimes B, Gutfleisch M et al: Serous pigment epithelial detachment in age-related macular degeneration: comparison of different treatments. Eye, 2009; 23: 2163-68 
16. Konstantinidis L, Ambresin A, Zografos L, Mantel I: Retinal pigment epithelium tears after intravitreal injection of ranibizumab for predominantly classic neovascular membranes secondary to age-related macular degeneration. Acta Ophthalmol, 2010; 88: 736-41

17. Arias L, Caminal JM, Rubio M et al: Retinal pigment epithelial tears after intravitreal bevacizumab injection for predominantly classic choroidal neovascularization. Eur J Ophthalmol, 2007; 17: 992-95

18. Gass JDM: Retinal pigment epithelial rip during krypton laser photocoagulation. Am J Ophthalmol, 1984; 98: 700-6

19. Krishan NR, Chandra SR, Stevens TS: Diagnosis and pathogenesis of retinal pigment epithelium tears. Am J Ophthalmol, 1985; 100: 698-707

20. Leitritz M, Gelisken F, Infoffen W et al: Can the risk of retinal pigment epithelium tears after bevacizumab treatment be predicted? An optical coherence tomography study. Eye, 2008; 22: 1504-7

21. Chan CK, Abraham P, Meyer CH et al: Optical coherence tomography - measured pigment epithelial detachment height as a predictor for retinal pigment epithelial tears associated with intravitreal bevacizumab injections. Retina, 2010; 30: 203-11
22. Gelisken F, Ziemssen F, Voelker M et al: Retinal pigment epithelial tears after single administration of intravitreal bevacizumab for neovascular age-related macular degeneration. Eye, 2009; 23: 694-702

23. Moroz I, Moisseiev J, Alhalel A: Optical coherence tomography predictors of retinal pigment epithelial tear following intravitreal bevacizumab injection. Ophthalmic Surg Lasers Imaging, 2009; 40: 570-75

24. Shiraki K, Kohno T, Ataka S et al: Thinning and small holes at an impending tear of a retinal pigment epithelial detachment. Graefes Arch Clin Exp Ophthalmol, 2001; 239: 430-36

25. Krejca M, Plewska A, Szmagala P et al: Outside stenting of the vein graft decreasese VEGF-A expression and induces significant down-regulation of VEGFR-1 in the intimal and medial layers after the re-endothelization period. Med Sci Monit, 2010; 16(3): 89-96

26. Czarkowska-Paczek B, Zendzian-Piotrowska M, Bartlomiejczyk I et al: Skeletal and heart muscle expression of PDGF-A and VEGF-A after an acute bout of exercise and endurance training in rats. Med Sci Monit, 2010; 16(5): BR147-153 